\title{
5G Vehicular Network for Smart Vehicles in Smart City: A Review
}

\author{
Hamzah U. Mustakim \\ Institut Teknologi Telkom Surabaya hamzah@ittelkom-sby.ac.id; \\ arekpanjen@gmail.com
}

\begin{abstract}
Smart vehicles is one of the innovation that can be used to increase the effectiveness of traffic in smart city as urban area and in the highway. In its development, smart vehicles not only require mobile communication services to ensure safety but also multimedia services for the passengers. These services must be integrated on intelligent transportation system. 5G vehicular network technology provides data communication services for intelligent transportation system via macrocells and smallcells to support smart vehicles which can drive at a maximum speed of $500 \mathrm{Km} /$ hour with multimedia services for passengers with data rates reaching the Gbps level. This paper will review 5G network architecture and emerging technologies that enabling vehicular network for smart vehicles in smart city.
\end{abstract}

Keywords: smart vehicles, smart city, 5G, microcell, smallcell, network, intelligent transportation system.

\section{Introduction}

Smart city is an innovation that developed in the last ten years by utilizing sensors, actuators, cloud technology as data and information centers connected to broadband communication networks. The concept of smart city will produce a variety of new service scenarios for the community so that it can change the style and way of life of people [1] and increase economic growth [10].

As a smart city enabler technology, 5G networks providing broadband communication services are the key technology that must be in place to serve data communication traffic from sensors to the cloud. There are three main scenarios in the 5G service: Enhanced Mobile Broadband (eMBB) for multimedia and mobile communication, then Ultra Reliable and Low Latency Communications (URLLC) can be used for transportation services and Massive Machine Type Communications (mMTC) for Internet of Things (IoT) applications that use many devices such as sensors and actuators. Development of 5G wireless technology will make cites smarter and more connected with smart items, smart vehicles and infrastructure (roads, buildings, etc.).

According to Colistra [2], in 2050, the global population is approaching 10 billion with a percentage of $70 \%$ being urban people. The concept of smart city is part of a new mechanism to build and organize the city and the environment within it with a variety of integrated internet connectivity to prepare for the future and a better quality of life.

In the 21st century, the concept of smart city can be translated into several main components which include smart economy, governance, society, science, technology, community life and the environment [4]. Smart cities are urban areas where there are various sectors that work together to achieve sustainable results through real-time information analysis by sharing specific information and operational technology systems [6]. The goal of the government to implement smart city is to improve operational effectiveness in meeting services to the public by utilizing technology and for the purpose of making decisions quickly and accurately in real-time [5]. Because of that we need a 
system that can integrate the main components and their derivatives, namely: smart house, smart vehicle, intelligent transportation system and others. Especially for smart vehicles, the development of this system leads to intelligent automobile systems or autonomous vehicles that are designed to work like the human brain [7]. The core of Smart vehicle is the internet of vehicle, which is an application of Internet of Things (IoT) technology in intelligent transportation system (ITS) [15], that lead to the development of vehicle to everything (V2X) including vehicle to vehicle $(\mathrm{V} 2 \mathrm{~V})$, vehicle to infrastructure (V2I), vehicle to pedestrian (V2P), vehicle to road (V2R) and vehicle to network (V2N) which require ultra high reliability [16].

\section{Vehicle to Everything Communication}

Vehicle to everything (V2X) communication is a technology and standard that enable a vehicle to communicate and interact with the user, road, infrastructure, pedestrian and network is shown in Figure 1 [19] [22].

\subsection{Vehicle to Vehicle Communication}

$\mathrm{V} 2 \mathrm{~V}$ communication services is designed for connected and autonomous vehicle need continuous access to vehicle's sensors to support advanced trajectory planning and high speed maneuver [20]. There are two trajectory planning schemes in V2V communication, first sort-term trajectory planning which is using data from its vehicle's sensor and long-term trajectory planning which is using another vehicles and infrastructure around. Therefor a connected vehicle can take dynamic decision safely depend on traffic situation [21].

\subsection{Vehicle to Pedestrian Communication}

V2P communication is aiming to improve the safety of pedestrians using cellular technology on smart phone. All of connected vehicles, cyclists and pedestrian will send information including speed, location and direction to the cloud server. These data is used to determine safety and hazard situation warning messages such as vehicle collision.

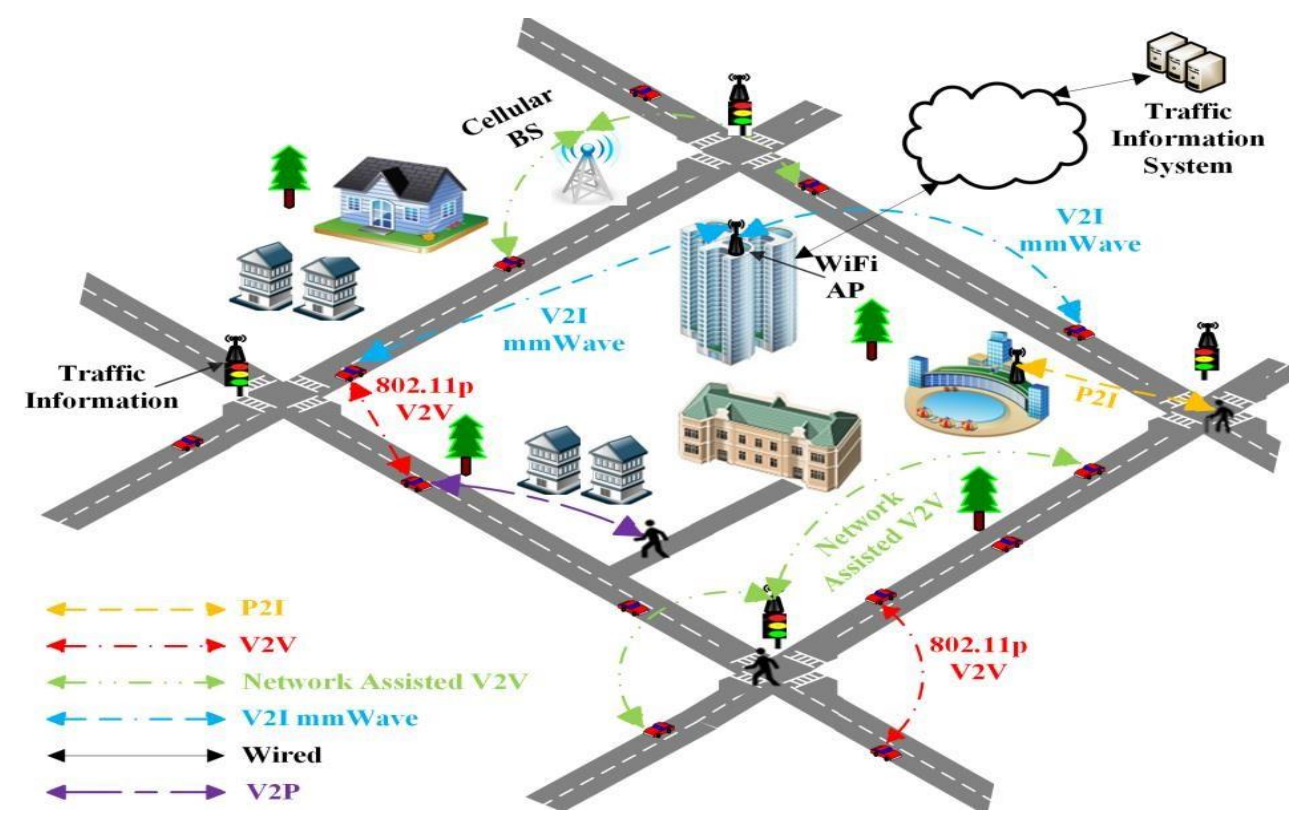

Figure 1. Kind of technologies for vehicle to everything communication [19]. 


\subsection{Vehicle to Infrasrukture Communication}

The main purpose of V2I is sharing information and data bidirectionally to the side road components such as traffic lights, street light, parking area, traffic camera, RFID, etc. the components that required by V2I communication are vehicle on-board unit (OBU), roadside unit (RSU) and safe communication channel. OBU is a radio transceiver for dedicated short range communication (DSRC) system, GPS, and provide communication with RSU. RSU made data transfer and message management to the vehicles.

\subsection{Intra Vehicle Communication}

Intra vehicle communication facilitating data transmission between vehicle components and vehicle sensors. This communication is important to make sure safety driving such as driver sensor which monitor driver condition and another vehicle sensors that monitor tire condition and braking system. These communication via wireless interconnection and ethernet.

\section{Vehicle Networking Communication Standards}

Development of smart vehicle network is based on all of networking system rather than one system including cellular system, short range communication system, WLAN, and satellite communication system. From these overall systems we can compose to be three core elements [15]: • Node: It is a vehicle network terminal element that used to collect and transmit data on vehicle, road, traffic and vehicle owner including GPS, On-board diagnostics (OBD), sensor, RFID, etc.

- Local Network: The network technology that used in a environment for traffic and roads to fulfil the requirements for vehicle communication.

- Internet of Things: A important component to built intelligent transportation system.

\subsection{Dedicated Short Range Communication.}

Dedicated Short Range Communication (DSRC) or IEEE 802.11p is a ad-hoc network for vehicle communication. A wireless access in vehicular environment which have good performance on delay while transmitting data in order to avoid vehicles collision [15] [17].

\subsection{Cellular Communication}

With large scale coverage area of Radio Access Network (RAN), Cellular communication technology has important role for machine to machine communication (M2M) as V2V application. 3GPP had defined standard communication for V2X based cellular network called C-V2X based 4G LTE (Figure 2) and future 5G. C-V2X will be good complement with DSRC technology [18].

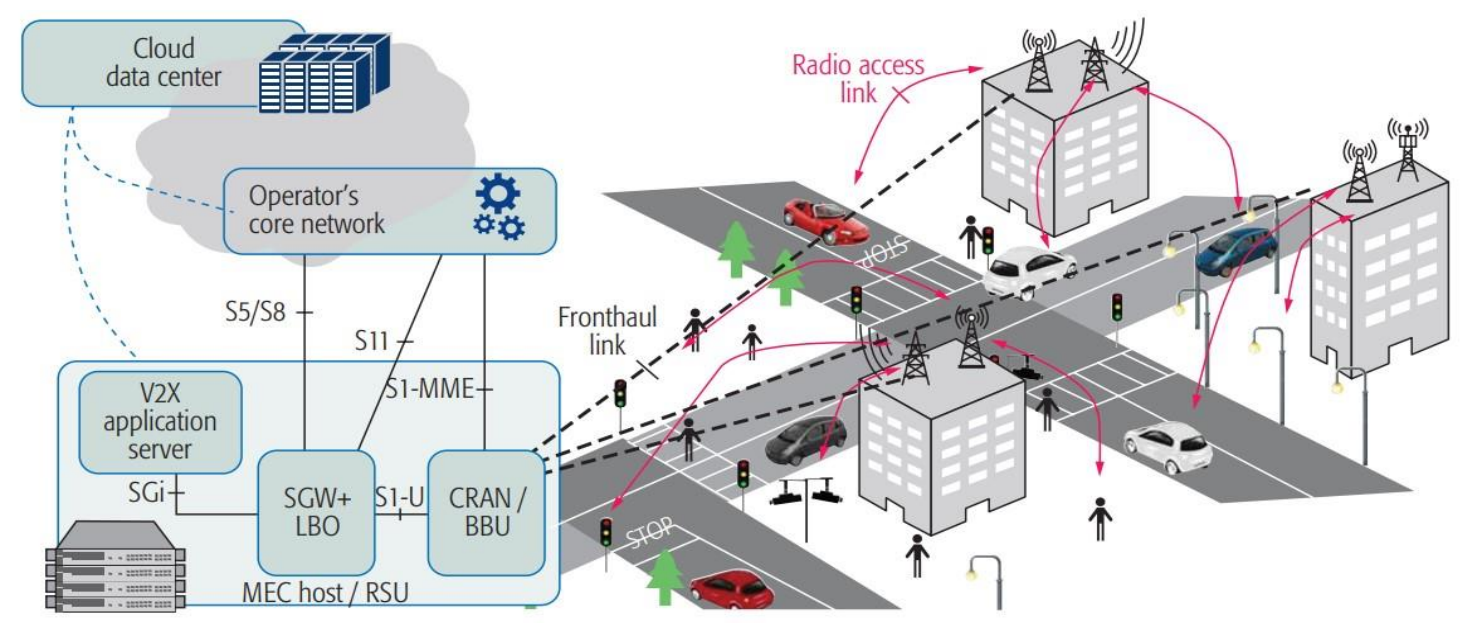

Figure 2. C-V2X based 4G network [18]. 


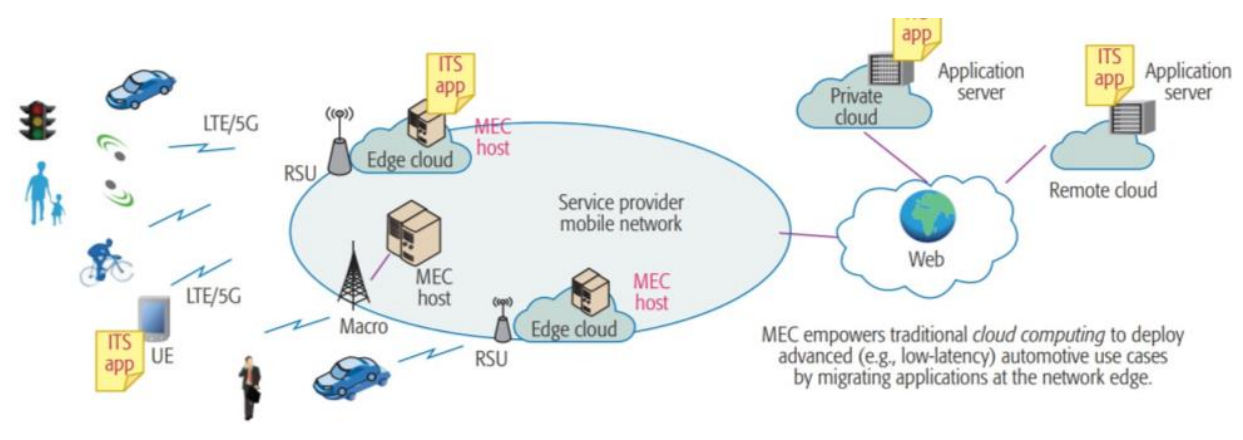

Figure 3. 5G network with MEC [18].

\subsection{Vehicle Ad-hoc Network}

Vehicle Ad-hoc Network (VANET) is a combination between DSRC and cellular technology or IEEE $802.11 \mathrm{p}$ within 4G LTE, that called LTE-V2X, support high speed mobility, reliable communication and better security level. LTE-V2X will use $802.11 \mathrm{p}$ frequency band for direct communication and have inertial navigation device that maintain positioning accuracy without GPS.

\section{5G Vehicullar Network}

There are three main scenarios in the $5 \mathrm{G}$ service, one of them is a service scenario used in data and multimedia communication applications with the main performance parameters at a data rate reaching up to 20Gbps on donwlink and 10Gbps at uplink [8]. The advantage of 5G technology is the ability to integrate and interconnect with existing networks using network function virtualization (NFV). The vehicullar network architecture in smart city services uses a centralized cloud system, distributed cloud and mobile cloud (Figure 3). In this network architecture there is the fog computing node that can provide mobile services for smart vehicles and multi-media applications for passenger. Part of the core of this architecture is the Visualization Plane Infrastructure which allows the formation of network visualization and function visualization [10].

Distributed clouds witch located at the edge of network infrastructure is called multi access edge computing (MEC) technology. The main purpose of MEC is fulfill low latency and high bandwidth requirements. As we mentioned above that vehicular communication require high level reliability $(99.99 \%)$ to maintain connection V2X [16].

Radio Access Network (RAN) technology which used for smart cars can be microcell, smallcell or microcell. For the use of microcell, you can use light poles on the side of the road with the advantage of being able to save infrastructure. The use of mmWave can significantly increase bandwidth and data rates. Smallcells are currently being developed using sensor technology and light emitting diodes (LEDs) [11]. Another thing to note is the 5G network can work at $4 \mathrm{G}$ network using non-stand alon scheme. To provide Gbps bit rate the use of fiber optic cable on the transport network, especially on the fronthaul which functions to send signals from the base band processing unit (BBU) to the antenna [13] [14]. Direct communication for V2V and V2I is called PC5 interface. PC5 using direct channel that not required $5 \mathrm{G}$ base station $(\mathrm{gNB})$.

\section{Conclusions}

The primary purposes of V2X communication technology is to improve driving safety and optimizing traffic in smart city. To achive that purposes required high reliability communication which can delivered by orchestration cellular technologi, 802.11p, DSRC, and utilization of MEC on $5 \mathrm{G}$ network technology. For the future research we need performance analysis (including troughput and hanover parameter) for each network which used in $5 \mathrm{G}$ vehicular network. 


\section{References}

1. E. Oproiu, M. Iordache, C. Patachia, C. Costea and I. Marghescu, "Development and implementation of a Smart City Use Case in a 5G mobile network's operator," 2017 25th Telecommunication Forum (TELFOR), Belgrade, 2017.

2. J. Colistra, "The Evolving Architecture of Smart Cities," 2018 IEEE International Smart Cities Conference (ISC2), Kansas City, MO, USA, 2018.

3. C. Colman-Meixner et al., "Deploying a Novel 5G-Enabled Architecture on City Infrastructure for UltraHigh Definition and Immersive Media Production and Broadcasting," in IEEE Transactions on Broadcasting, vol. 65, no. 2, pp. 392-403, June 2019.

4. E. Mardacany, "Smart cities characteristics: importance of buit environments components," IET Conference on Future Intelligent Cities, London, 2014.

5. M. Abu-Matar and R. Mizouni, "Variability Modeling for Smart City Reference Architectures," 2018 IEEE International Smart Cities Conference (ISC2), Kansas City, MO, USA, 2018.

6. Gartner, Inc., "Market Guide for Smart City Operations Management Platforms and Ecosystems”, 2015.

7. M. Karaduman and H. Eren, "Smart driving in smart city," 2017 5th International Istanbul Smart Grid and Cities Congress and Fair (ICSG), Istanbul, 2017.

8. Afif Osseiran, "5G Mobile and Wireless Communications Technology", Cambridge University Press, 2016.

9. M. A. Habibi, M. Nasimi, B. Han and H. D. Schotten, "A Comprehensive Survey of RAN Architectures Toward 5G Mobile Communication System," in IEEE Access, vol. 7, pp. 70371-70421, 2019.

10. A. Karadimce and N. Marina, "Smart Mobile City Services in the 5G Era," 2018 10th International Congress on Ultra Modern Telecommunications and Control Systems and Workshops (ICUMT), Moscow, Russia, 2018.

11. B. Gholampooryazdi, H. Hämmäinen, S. Vijay and A. Savisalo, "Scenario planning for 5G light poles in smart cities," 2017 Internet of Things Business Models, Users, and Networks, Copenhagen, 2017.

12. IMT Vision, "Framework and overall objectives of the future development of IMT for 2020 and beyond", 2015.

13. P. T. Dat, A. Kanno, N. Yamamoto and T. Kawanishi, "Seamless Convergence of Fiber and Wireless Systems for 5G and Beyond Networks," in Journal of Lightwave Technology, vol. 37, no. 2, pp. 592-605, 15 Jan.15, 2019.

14. K. Zeb, X. Zhang and Z. Lu, "High Capacity Mode Division Multiplexing Based MIMO Enabled All-Optical Analog Millimeter-Wave Over Fiber Fronthaul Architecture for 5G and Beyond," in IEEE Access, vol. 7, pp. 89522-89533, 2019.

15. Y. Yang and K. Hua, "Emerging Technologies for 5G-Enabled Vehicular Networks," in IEEE Access, vol. 7, pp. 181117-181141, 2019.

16. D. Garcia-Roger et al., "5G Functional Architecture and Signaling Enhancements to Support Path Management for eV2X," in IEEE Access, vol. 7, pp. 20484-20498, 2019.

17. Chang K H. Wireless communications for vehicular safety[J]. IEEE Wireless Communications, 2015, 22(1): 6-7.

18. F. Giust et al., "Multi-Access Edge Computing: The Driver Behind the Wheel of 5G-Connected Cars," in IEEE Communications Standards Magazine, vol. 2, no. 3, pp. 66-73, SEPTEMBER 2018.

19. H. Ullah, N. Gopalakrishnan Nair, A. Moore, C. Nugent, P. Muschamp and M. Cuevas, "5G Communication: An Overview of Vehicle-to-Everything, Drones, and Healthcare Use-Cases," in IEEE Access, vol. 7, pp. 37251-37268, 2019.

20. I. Mavromatis, A. Tassi, R. J. Piechocki, and A. Nix, “Efficient V2V communication scheme for 5G mmWave hyper-connected CAVs," in Proc. IEEE Int. Conf. Commun., May 2018, pp. 1-6.

21. N. Lu, N. Cheng, N. Zhang, X. Shen, and J. W. Mark, “Connected vehicles: Solutions and challenges," IEEE Internet Things J., vol. 1, no. 4, p 5G Evolution: A View on 5G Cellular Technology Beyond 3GPP Release 15 p. 289-299, Aug. 2014.

(C) 2019 by the authors. Submitted for possible open access publication under the terms and conditions of the Creative Commons Attribution (CC BY) license (http://creativecommons.org/licenses/by/4.0/). 
Complete 2020, Vol. 1, No. 1, doi.org/10.52435/complete.v1i1.44 